\title{
BMJ Open Association of trajectory of body mass index with knee pain risk in Japanese middle-aged women in a prospective cohort study: the Japan Nurses' Health Study
}

\author{
Ayumi Ito, ${ }^{1}$ Kunihiko Hayashi (D) , ${ }^{1}$ Shosuke Suzuki, ${ }^{2,3}$ Yuki Ideno, ${ }^{4}$ \\ Takumi Kurabayashi, ${ }^{5}$ Toru Ogata, ${ }^{6}$ Atsushi Seichi, ${ }^{7}$ Masami Akai (D) , ${ }^{8}$ \\ Tsutomu Iwaya ${ }^{9}$
}

To cite: Ito A, Hayashi $\mathrm{K}$ Suzuki S, et al. Association of trajectory of body mass index with knee pain risk in Japanese middle-aged women in a prospective cohort study: the Japan Nurses' Health Study. BMJ Open 2020;10:e033853. doi:10.1136/ bmjopen-2019-033853

- Prepublication history for this paper is available online. To view these files, please visit the journal online (http://dx.doi org/10.1136/bmjopen-2019033853).

Received 18 December 2019 Revised 27 December 2019 Accepted 09 January 2020

Check for updates

(C) Author(s) (or their employer(s)) 2020. Re-use permitted under CC BY-NC. No commercial re-use. See rights and permissions. Published by BMJ.

For numbered affiliations see end of article.

Correspondence to Dr Kunihiko Hayashi; khayashi@gunma-u.ac.jp

\section{ABSTRACT}

Objectives To investigate whether body mass index (BMI) trajectory, lifestyle and reproductive factors are associated with knee pain risk among middle-aged women.

Design Prospective study of the Japan Nurses' Health Study (JNHS).

Setting The JNHS investigates the health of female nurses in Japan. Biennial follow-up questionnaires are mailed to the participants.

Participants The 7434 women aged over 40 years who responded to the 10-year self-administered follow-up questionnaire.

Primary outcome measure Self-reported knee pain at the 10-year follow-up was the primary outcome. We analysed BMI (normal or overweight) trajectory data from a baseline survey to the 10-year follow-up survey using group-based trajectory modelling. Exposure measurements were BMI trajectory, BMl at age 18 years, lifestyle variables and reproductive history.

Results BMI trajectories from baseline to the 10-year follow-up were divided into four groups: remained normal, remained overweight, gained weight or lost weight. At the 10-year follow-up, 1281 women (17.2\%) reported knee pain. Multivariable logistic regression analysis revealed that compared with the remained normal group, multivariableadjusted ORs $(95 \% \mathrm{Cl})$ of knee pain were 1.93 (1.60 to 2.33 ) for the remained overweight group, 1.60 (1.23 to 2.08) for the gained weight group and 1.40 ( 0.88 to 2.21) for the lost weight group. The attributable risk percent $(95 \% \mathrm{Cl})$ of the remained overweight group was $48.1 \%(37.3 \%$ to $57.0 \%)$ compared with the reference group of remained normal. Alcohol intake at baseline was significantly associated with knee pain.

Conclusions The lost weight group had a lower risk than the remained overweight group and the gained weight group and did not carry statistically significant risks for knee pain. Weight reduction and maintaining a normal BMI in middle age was important for preventing knee pain in women.

\section{INTRODUCTION}

Knee pain, along with lower back pain, is one of the most problematic musculoskeletal

\section{Strengths and limitations of this study}

- The longitudinal cohort study design provides data regarding 10-year trajectory in body mass index.

- The Japan Nurses' Health Study, a large cohort study of female nurses, enabled comprehensive analysis, drawing on rich and accurate information regarding healthcare, reproductive health and lifestyle.

- We selected knee pain as the primary outcome, whereas many studies have investigated risk factors for knee osteoarthritis and insufficiently examined risk factors for knee pain.

- Participants were asked to provide their height and weight via self-assessment; we calculated body mass index on the basis of this information.

- We were unable to obtain information regarding the severity or duration of knee pain or the presence of knee pain at baseline; we therefore excluded women who had been diagnosed with knee osteoarthritis prior to the baseline survey.

disorders, and impedes function and affects movement and daily life. ${ }^{1-4}$ Knee pain is caused by the age-related weakness of the knee joint, or excessive load on a knee joint, as with knee osteoarthritis $(\mathrm{KOA}) .^{5}{ }^{6}$ An example of excessive load on a knee joint is one's own body weight. Furthermore, the load of long-term body weight has a great influence on a knee. ${ }^{7}$ Although it is necessary to evaluate changes in body weight over time, when we think of knee pain, few studies have investigated the association of the trajectory of body weight and knee pain. There is a sex difference in the prevalence of knee pain: more women experience knee pain than men. ${ }^{4}$ Considering this sex difference in the prevalence of knee pain, it is important to examine associations of knee pain with 
reproductive and lifestyle factors among women. Moreover, several female reproductive factors were associated with BMI in a prior study, ${ }^{8}$ so the association between BMI trajectory and knee pain merits investigation.

KOA is a disease that is typically accompanied by severe knee pain. Many publications have reported risk factors for KOA. Ageing and high BMI are well-known factors; other lifestyle (eg, smoking, alcohol drinking habits and sleep duration) and female reproductive factors are also thought to be associated with KOA. ${ }^{9-15}$ Some studies have reported that menopausal status, parity and age at menarche are associated with KOA. ${ }^{15}$ However, a systematic review reported no significant associations between female hormonal characteristics and KOA. ${ }^{16}$ Risk factors for knee pain in male and female working populations were found to be similar to risk factors for KOA in those same populations. ${ }^{17}$

It has been reported that KOA appears after chronic knee symptoms. ${ }^{18}$ We examined knee pain as a primary outcome, because knee pain helps to identify early stage $\mathrm{KOA}$, and pain may appear before the diagnosis of musculoskeletal diseases. We conducted a prospective cohort study on the association between BMI trajectory in middle age, lifestyle, reproductive history and knee pain in middle-aged women.

\section{METHODS}

\section{Study population}

The Japan Nurses' Health Study (JNHS), which started in 2001, is a prospective cohort study to investigate the effects of lifestyle and healthcare practices on the health of female nurses in Japan. Participants were female public health nurses, midwives, registered nurses and assistant nurses aged 25 years or older at the time of registration. The JNHS continues through follow-up surveys conducted once every 2years by a postal self-administered questionnaire. ${ }^{19} 20$ The subjects of the current study included 10667 women over the age of 40 years at the 10-year follow-up who responded to the 10-year follow-up surveys. We excluded 142 women who had diagnosed KOA on the baseline survey, 575 women who became pregnant at least once during the 10-year follow-up period and 2516 women who had not provided their body weight or height for the calculation of BMI between the baseline survey to the 10 years. The remaining 7434 women were analysed (figure 1).

\section{Outcomes and covariates}

Knee pain on the 10-year follow-up surveys was the primary outcome. Participants were asked if they were currently experiencing knee pain, to which they responded yes or no. We also collected data regarding age, female reproductive factors and menopausal status, and parity on the 10-year follow-up questionnaire.

The other covariate data used from the baseline survey were lifestyle factors including smoking status, alcohol intake and sleep duration, and female reproductive factor including age at menarche and body weight at age 18 years

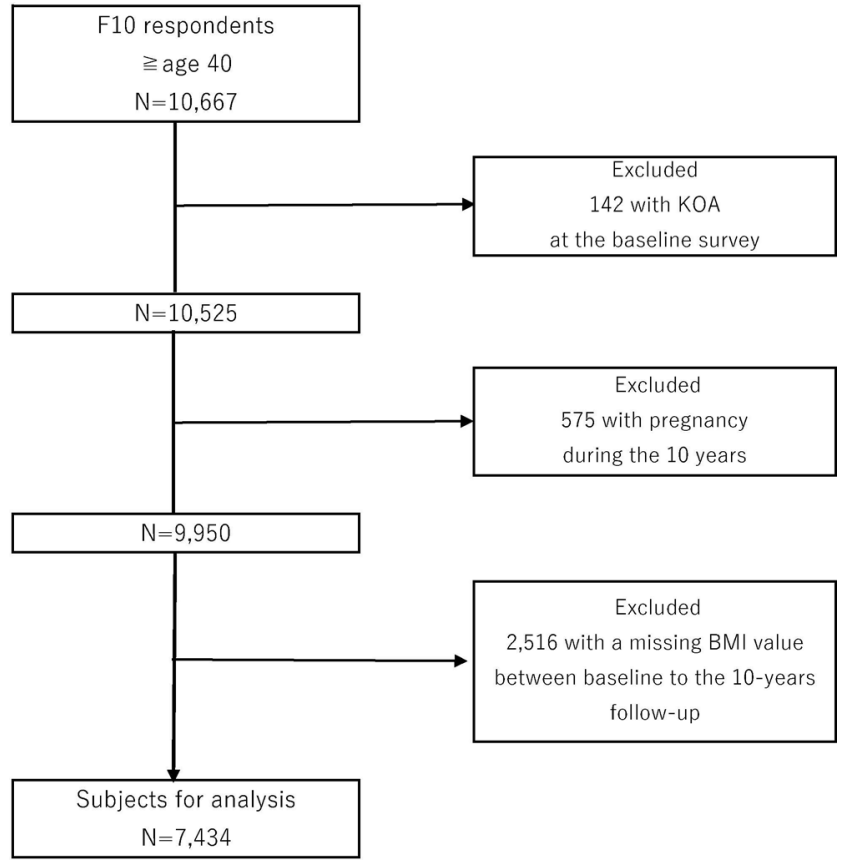

Figure 1 Selection of subjects for our analysis from the Japan Nurses' Health Study. Of the 10667 women over 40 years old who responded to the 10-year follow-up (F10) survey, a total of 7434 were included in our analysis. BMI, body mass index; KOA, knee osteoarthritis.

(included as BMI at age 18 years). To identify the BMI trajectory, weight and height from the baseline survey to the 10-year follow-up survey were used. The participants were listed as either never-smokers or ever-smokers (including former smokers and current smokers). Alcohol intake was defined by intake frequency: non-drinkers were those who never drank or drank one to two times per month, light drinkers were those who drank one to two times per week, moderate drinkers were those who drank three to six times per week and heavy drinkers were those who drank every day. The participants were divided into three groups based on average sleep duration, regardless of shift pattern: short ( $<6$ hours per night), moderate ( $6-<7$ hours per night) and long ( $\geq 7$ hours per night). For menopausal status, women were classified as premenopausal or postmenopausal; notably, postmenopausal included those who were undetermined (ie, those undergoing hormone replacement therapy and those in whom it could not be determined whether they were postmenopausal due to a transitional period). Age at menarche was categorised by tertiles as early ( $\leq 11$ years old), moderate (12 years old) or late ( $\geq 13$ years old). We calculated BMI using the women's self-reported height and weight at age 18 years and between the baseline survey and the 10-year follow-up survey. We converted the continuous BMI variable into either normal or overweight prior to drawing the BMI trajectories. BMI $<25 \mathrm{~kg} /$ $\mathrm{m}^{2}$ was regarded as normal, and BMI $\geq 25$ was regarded as overweight for all time points. We defined BMI trajectory as the change in BMI from the baseline survey to the 10-year follow-up survey. 


\section{Statistical analysis}

Data analyses were performed using SAS statistical software, V.9.4. Group-based trajectory modelling was used to analyse BMI trajectory for indicating the longitudinal changes using PROC TRAJ in SAS. ${ }^{21-23}$ This method is one of finite mixture modelling, where some groups are made around an individual BMI trace.

To examine differences between subjects with and without knee pain, $\chi^{2}$ tests were used for nominal variables, and Mann-Whitney $U$ tests were used for ordinal variables. A logistic regression analysis was conducted to explore the associations between knee pain and BMI trajectory, lifestyle and reproductive factors. We selected all variables for logistic regression analysis (full model) regardless of univariate relationships. We calculated multivariableadjusted ORs and 95\% CIs, adjusting for age at the 10-year follow-up survey, and for BMI trajectory, smoking, alcohol intake, sleep duration, menopausal status, parity, age at menarche and BMI at age 18 years. The attributable risk percent of the remained overweight group was estimated using multivariable-adjusted OR for BMI trajectory, when the reference group was the other groups.
For sensitivity analyses, the analysis excluded women who had self-reported having cancer due to the possible influence on BMI, and the analysis with multiple imputation for the missing BMI data from the 2-year to 8-year follow-up survey was conducted to confirm the results of primary analyses. ${ }^{24}$

The results were regarded as statistically significant when two-tailed $\mathrm{p}$ values were less than 0.05 .

\section{Ethical consideration}

Participants were informed of the purpose and procedures of the JNHS. All participants signed consent sheets when they completed the baseline survey.

\section{Patient and public involvement}

Patients and the public were not involved this study. The results will not be presented at conferences. The announcement of the publication is expected to be sufficient. We send annual newsletters to all study participants; these newsletters include information regarding JNHS papers that were published in the previous year.

Table 1 Age, BMI trajectories, lifestyle variables and reproductive variables for the population of 7434 women

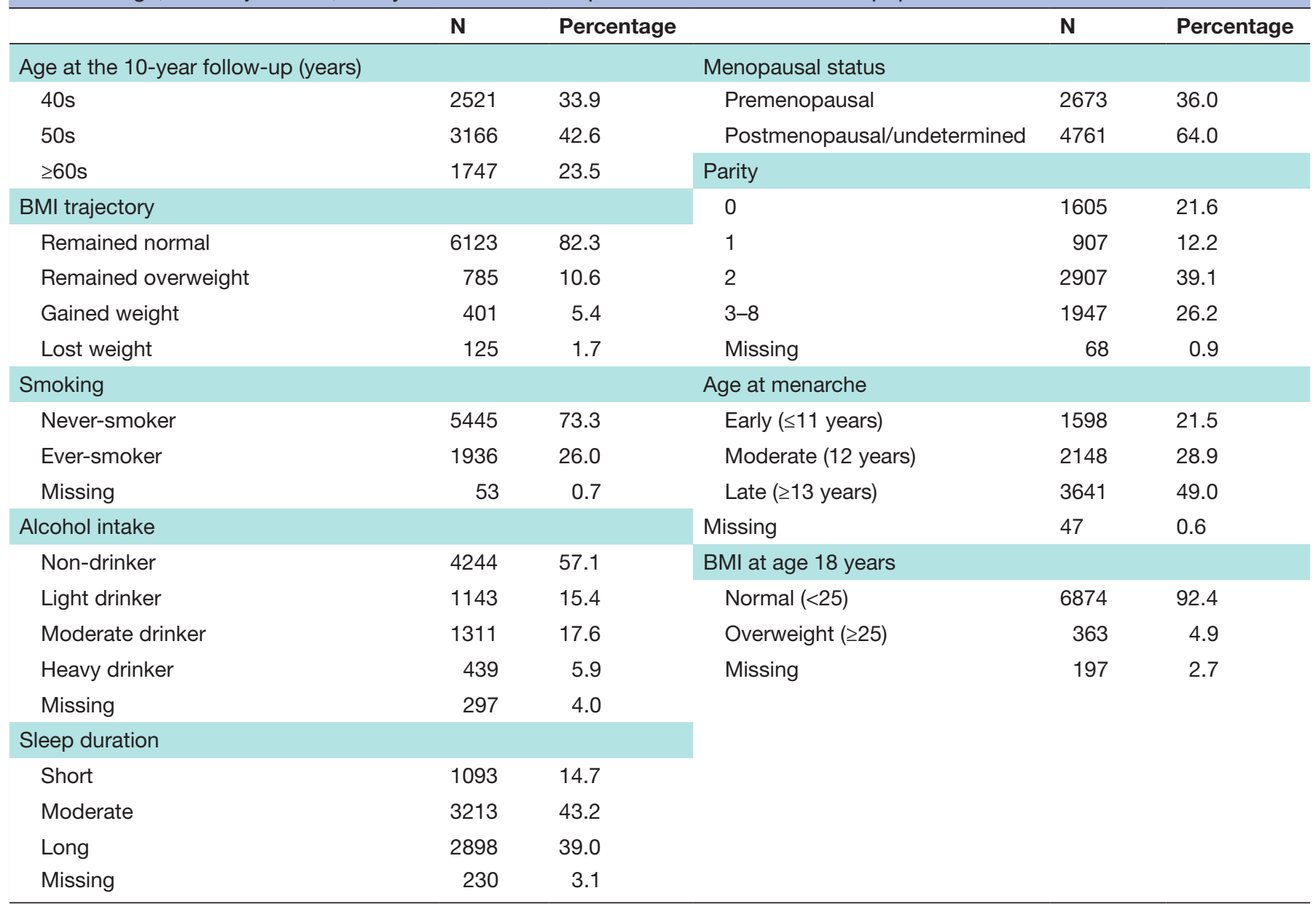

BMI trajectory: BMI from the baseline to the 10-year follow-up survey.

$\mathrm{BMI}$, body mass index. 


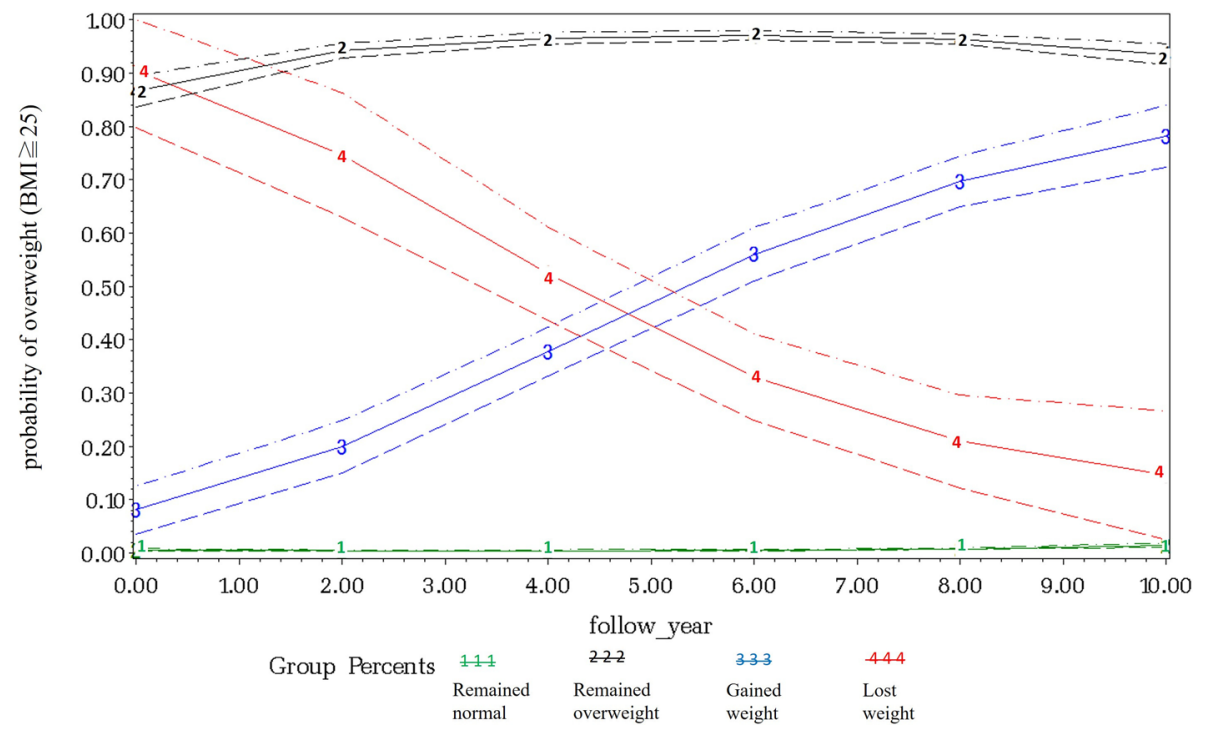

Figure 2 Trajectories of body mass index (BMI) changes. BMI was converted to binary data: BMI $\geq 25$ as overweight was 1.0 , and $\mathrm{BMI}<25$ as normal was 0.0 . Ninety-five per cent Cls are shown as a dashed line. Overall, $82.3 \%$ remained normal weight; $10.6 \%$ remained overweight; $5.4 \%$ gained weight; $1.7 \%$ lost weight.

\section{RESULTS}

\section{Characteristics of the study population}

The characteristics of the study population are shown in table 1 . The mean (SD) age was 53.5 (7.7) years. The mean BMI at age 18 was 21.1 (2.3), and the mean BMI at the baseline survey was 21.8 (2.8). The percentage of those who were overweight based on BMI at the baseline survey was $11.9 \%$, while $88.1 \%$ had a normal BMI. At the 10-year follow-up survey, the mean BMI was 22.2 (3.2), overweight BMI was $16.0 \%$ and normal BMI was $84.0 \%$. Regarding the four BMI trajectory groups, there were $6123(82.3 \%)$ women in the remained normal group, 785 $(10.6 \%)$ in the remained overweight group, $401(5.4 \%)$ in the gained weight group and $125(1.7 \%)$ in the lost weight group (figure 2). The mean (SD) sleep duration was $6.5(0.9)$ hours per night. The mean (SD) age at menarche was 12.6 (1.3) years. The population included $2673(36.0 \%)$ premenopausal women and 4761 (64.0\%) postmenopausal women.

\section{Prevalence of knee pain and musculoskeletal diseases}

A total of $1281(17.2 \%)$ women with knee pain at the 10-year follow-up survey were identified in the study population (table 2). Table 2 shows $p$ values from the $\chi^{2}$ tests for nominal variables and from Mann-Whitney $\mathrm{U}$ tests for ordinal variables to examine the differences between respondents with and without knee pain.

\section{Factors associated with knee pain}

Table 2 also shows the associations between knee pain and potential risk factors from multivariable logistic regression. Age at the time of the 10-year follow-up survey was significantly associated with risk of knee pain. Multivariable-adjusted ORs (95\% CIs) were 1.29 (1.03 to 1.61 ) and 1.57 (1.21 to 2.04) for women between the age of 50-59 years and age 60 years or older, respectively, compared with women between 40 years and 49 years.
Multivariable-adjusted ORs (95\% CIs) were 1.93 (1.60 to 2.33 ), 1.60 (1.23 to 2.08) and 1.40 (0.88 to 2.21) for remained overweight, gained weight and lost weight groups, respectively, compared with women in the remained normal group. The attributable risk per cent (ie, excess risk fraction) (95\% CI) of the remained overweight group was $48.1 \%$ ( $37.3 \%$ to $57.0 \%$ ), $27.5 \%$ ( $-17.0 \%$ to $55.1 \%)$ and $16.8 \%$ ( $-12.3 \%$ to $38.4 \%$ ) compared with the reference groups of remained normal, lost weight and gained weight groups, respectively. The association between BMI at age 18 years and knee pain was not statistically significant (multivariable-adjusted $\mathrm{OR}=1.28$, $95 \%$ CI 0.98 to 1.68 ).

The multivariable-adjusted OR for experiencing knee pain was 1.37 (95\% CI 1.06 to 1.77) for heavy drinker compared with non-drinker. There were no statistically significant associations between knee pain and smoking, sleep duration, menopausal status, parity or age at menarche.

We conducted sensitivity analyses, multivariableadjusted ORs (95\% CIs) excluded women who had self-reported having cancer were 2.09 (1.70 to 2.56), 1.62 (1.22 to 2.15) and 1.40 (0.83 to 2.37) for the remained overweight, gained weight and lost weight groups, respectively, compared with women in the remained normal group. In another sensitivity analysis, the multiple imputation for the missing BMI data from the 2-year to 8-year follow-up survey showed that multivariable-adjusted ORs (95\% CIs) were 2.11 (1.78 to 2.49 ), 1.62 (1.27 to 2.06 ) and 1.23 (0.93 to 1.62 ) for the remained overweight, gained weight and lost weight groups, respectively, compared with women in the remained normal group.

According to these sensitivity analyses, we confirmed there were no significant differences in the other resulting ORs of BMI trajectory using logistic regression. 
Table 2 Prevalence and multivariable-adjusted ORs for knee pain on the 10-year follow-up survey

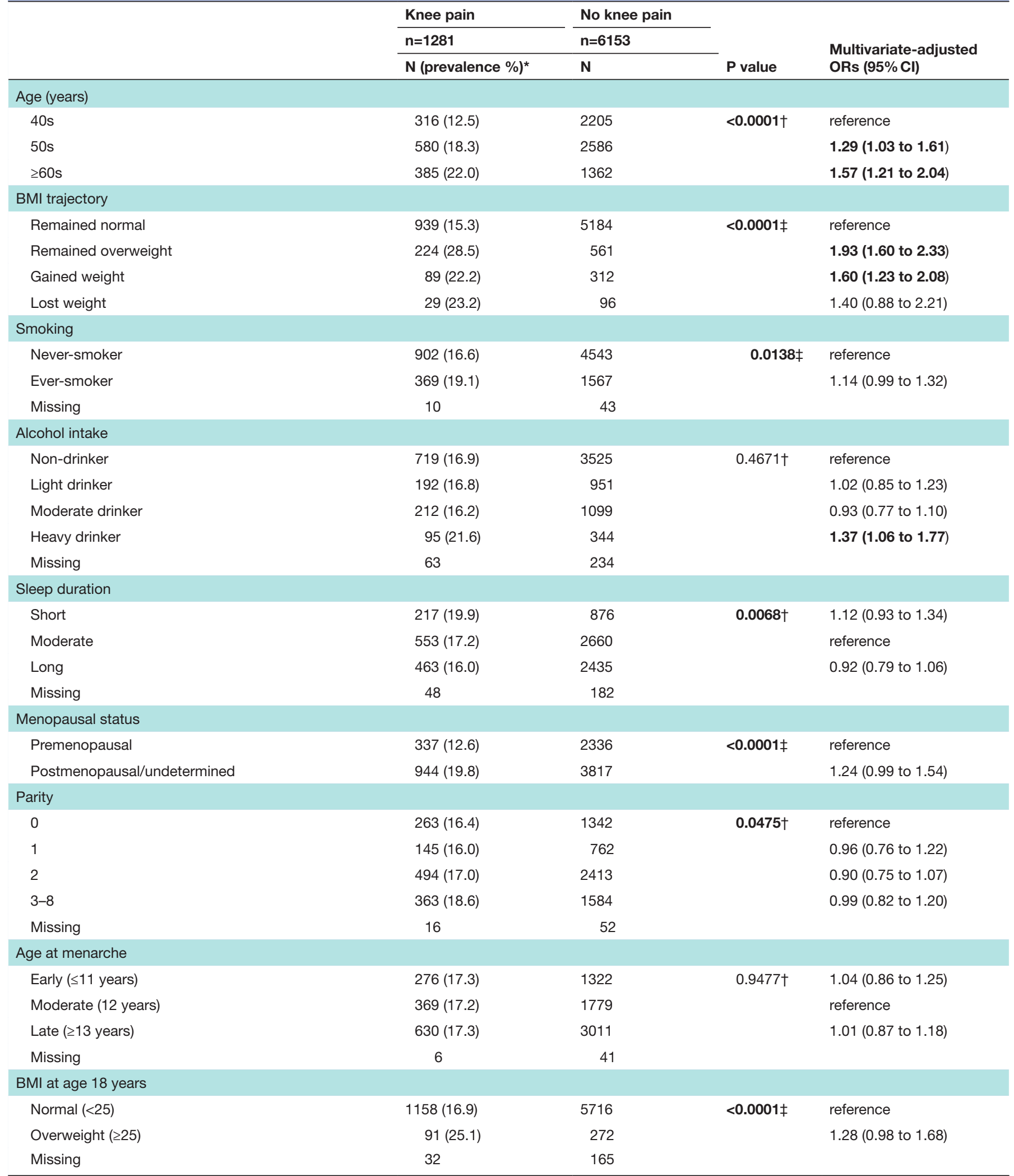

Continued 


\begin{tabular}{llll} 
Knee pain & No knee pain & & \\
\cline { 1 - 1 }$n n=1281$ & $N$ P value & $\begin{array}{l}\text { Multivariate-adjusted } \\
\text { ORs }(95 \% \mathrm{Cl})\end{array}$
\end{tabular}

Multivariable-adjusted ORs $(95 \% \mathrm{Cl})$, adjusted for age at the 10-year survey, BMI trajectory, smoking, alcohol intake, sleep duration, menopausal status, parity, age at menarche and $\mathrm{BMI}$ at age 18 years.

BMI trajectory: BMI from baseline to the 10-year follow-up survey.

Bold values: statistically significant.

*Prevalence of knee pain (\%).

†Mann-Whitney $U$ test.

$\neq \chi^{2}$.

$\mathrm{BMI}$, body mass index.

\section{DISCUSSION}

We found that BMI trajectory was a predictive factor for knee pain. Remaining overweight increased the risk of knee pain. It is known that high BMI is associated with knee pain or $\mathrm{KOA}^{9}$ and our results were partially in agreement with prior studies. We found that remaining overweight was associated with a 1.9 times increased risk compared with those who remained at a normal weight. The risk of knee pain was increased approximately 1.6 times when the women who were normal weight became overweight. The women with normal BMI should continue to control her weight in order to maintain the low risk for knee pain. However, if overweight women reduced their weight over 10 years, they were able to decrease the risk of knee pain by $27.5 \%$. Furthermore, remaining overweight group and gained weight group carried statistically significant risks for knee pain; however, lost weight group did not. This BMI trajectory showed the need to reduce the weight and maintain a normal BMI to help prevent knee pain. There are some studies that have described weight change and its influence on knee pain. ${ }^{925}{ }^{26}$ Our study showed that a dynamic BMI change was related to knee pain. We found that there was no statistically significant association between BMI at age 18 years and knee pain in middle age, and BMI trajectory in middle age was a much stronger predictor of knee pain compared with BMI at age 18 years, although these results were different from a prior study in men. ${ }^{27}$ This phenomenon of knee pain may be caused by loading on the knee joint. The mechanisms of the association between obesity and knee pain constitute a combination of mechanical, structural, metabolic and behavioural changes. ${ }^{28}$ Obesity leads to a loss of muscle mass and strength, as well as the accumulation of fat tissue; these factors together contribute to knee joint compression. ${ }^{29}$ Conversely, weight loss may improve physical activity level, increase muscle strength and reduce knee pain-related disability. ${ }^{30}$ Our findings suggest that major weight gain or the presence of an overweight status for a long period in middle age are probable risk factors for knee pain. In addition, weight loss and maintenance of normal weight may prevent the development of knee pain. Musculoskeletal pain might impact healthrelated quality of life. ${ }^{31}$ Therefore, the continuous control of body weight in middle age may be effective to prevent knee pain and to maintain quality of life.
The present study had several strengths. First, the present study was a longitudinal cohort study, such that a 10-year trajectory in BMI could be examined. Second, because the JNHS comprises a large cohort study of female nurses, we were able to conduct a comprehensive analysis, drawing on rich and accurate information regarding healthcare, reproductive health and lifestyle. Third, we selected knee pain as the primary outcome, whereas many studies have investigated risk factors for KOA and insufficiently examined risk factors for knee pain. Knee pain is a prodromal symptom of KOA; it helps us to identify the risk of KOA. However, the present study had two limitations. First, participants were asked to provide their height and weight, which was a self-assessment. We calculated BMI on the basis of this information. Second, we were unable to obtain information regarding the severity or duration of knee pain or the presence of knee pain at baseline; if we had this information, we could assess the incidence of knee pain from baseline among Japanese female nurses in middle age. However, we excluded women who had been diagnosed with KOA prior to the baseline survey.

The prevalence of knee pain in the present study among Japanese nurses (17.2\%) was higher than the prevalence of extremity joint pain previously found among the general population of Japanese women $(7.0 \%){ }^{32}$ In previous studies involving nurses, the prevalence of knee symptoms (including ache, pain and discomfort) ranged from 10.6\% to $24.7 \%$ in the USA, Australia, Korea and Estonia. ${ }^{33-36}$ Nurses worldwide are clearly in at-risk settings for knee symptoms, and consideration must be given to nurses' knee pain. One of the limitations of the present study was the composition of the study population. Nurses may be exposed to different risk factors, such as manually handling patients, hard physical work and high mental pressure because of their working circumstances. ${ }^{37}$ Therefore, our findings regarding prevalence may not be generalisable to the general population. However, there is no reason to suspect that the general population of women would differ in the association between the risk of knee pain and BMI trajectory.

Heavy drinkers had a risk for 10 years later knee pain than non-drinkers in this study, whilst the association between alcohol intake and KOA also had shown in a previous study. ${ }^{10}$ There is no convincing explanation about the 
mechanism of the association between alcohol drinking habit and knee pain.

In conclusion, the lost weight group had a lower risk than the remained overweight group and the gained weight group. From our longitudinal observational study of the 10-year trajectory in BMI, we found that weight reduction and maintaining a normal BMI in middle age were important for preventing knee pain in women. Further studies are needed to indicate the effectiveness of losing weight and maintaining a normal BMI in the prevention of knee pain.

\section{Author affiliations}

${ }^{1}$ Unit of International and Community Health Sciences, Graduate School of Health Sciences, Gunma University, Maebashi, Japan

${ }^{2}$ Faculty of Medicine, Gunma University, Maebashi, Japan

${ }^{3}$ Eco-Health Research Group, Isesaki, Japan

${ }^{4}$ Center for Mathematics and Data Science, Gunma University, Maebashi, Japan ${ }^{5}$ Department of Obstetrics \& Gynecology, Niigata City General Hospital, Niigata, Japan

${ }^{6}$ Center for Sport Science and Health Promotion, Department of Rehabilitaion for the Movement Functions, National Rehabilitation Center for Persons with Disabilities, Tokorozawa, Japan

${ }^{7}$ Department of Orthopaedic Surgery, Mitsui Memorial Hospital, Chiyoda-ku, Japan ${ }^{8}$ Graduate School, International University of Health and Welfare Kohokai Group, Minato-ku, Japan

${ }^{9}$ Nagano University of Health and Medicine, Nagano, Japan

Acknowledgements The authors would like to thank Drs Yukiko Miyazaki, Ayumi Kobayashi and Kazue Nagai for their advice.

Contributors Al analysed the data and drafted the report. KH designed and initiated the study. Al, KH, SS and YI contributed to the interpretation and discussion of the data and writing of the manuscript. All authors approved the final draft to be published and have agreed to be accountable for all aspects of the work, ensuring that questions related to the accuracy or integrity of any part of the work are appropriately investigated and resolved.

Funding This work was partly supported by a Japanese Society for Musculoskeletal Medicine Research Grant, as well as JSPS KAKENHI Grant Number $18 \mathrm{H} 04069$.

Competing interests None declared.

Patient consent for publication Not required.

Ethics approval The JNHS study protocol was approved by the institutional review board of the Gunma University (Approval No. 101, study protocol=JNHS0101).

Provenance and peer review Not commissioned; externally peer reviewed.

Data availability statement All data relevant to the study are included in the article.

Open access This is an open access article distributed in accordance with the Creative Commons Attribution Non Commercial (CC BY-NC 4.0) license, which permits others to distribute, remix, adapt, build upon this work non-commercially, and license their derivative works on different terms, provided the original work is properly cited, appropriate credit is given, any changes made indicated, and the use is non-commercial. See: http://creativecommons.org/licenses/by-nc/4.0/.

\section{ORCID iDs}

Kunihiko Hayashi http://orcid.org/0000-0003-4648-3936

Masami Akai http://orcid.org/0000-0002-9145-7158

\section{REFERENCES}

1 Alexopoulos EC, Tanagra D, Detorakis I, et al. Knee and low back complaints in professional Hospital nurses: occurrence, chronicity, care seeking and absenteeism. Work 2011;38:329-35.

2 Agaliotis M, Mackey MG, Jan S, et al. Burden of reduced work productivity among people with chronic knee pain: a systematic review. Occup Environ Med 2014;71:651-9.
3 Coggon D, Ntani G, Palmer KT, et al. The CUPID (cultural and psychosocial influences on disability) study: methods of data collection and characteristics of study sample. PLoS One 2012; 7:e39820.

4 Andersen RE, Crespo CJ, Ling SM, et al. Prevalence of significant knee pain among older Americans: results from the third National health and nutrition examination survey. J Am Geriatr Soc 1999;47:1435-8.

5 Ding C, Cicuttini F, Blizzard L, et al. A longitudinal study of the effect of sex and age on rate of change in knee cartilage volume in adults. Rheumatology 2007;46:273-9.

6 Mezhov V, Ciccutini FM, Hanna FS, et al. Does obesity affect knee cartilage? A systematic review of magnetic resonance imaging data. Obes Rev 2014;15:143-57.

7 Dawson J, Juszczak E, Thorogood M, et al. An investigation of risk factors for symptomatic osteoarthritis of the knee in women using a life course approach. J Epidemiol Community Health 2003;57:823-30.

8 Newby PK, Dickman PW, Adami H-O, et al. Early anthropometric measures and reproductive factors as predictors of body mass index and obesity among older women. Int J Obes 2005;29:1084-92.

9 Felson DT, Zhang Y, Hannan MT, et al. Risk factors for incident radiographic knee osteoarthritis in the elderly: the Framingham study. Arthritis Rheum 1997;40:728-33.

10 Muthuri SG, Zhang W, Maciewicz RA, et al. Beer and wine consumption and risk of knee or hip osteoarthritis: a case control study. Arthritis Res Ther 2015;17:23.

11 Zhang Y, Zeng C, Wei J, et al. Associations of cigarette smoking, betel quid chewing and alcohol consumption with high-sensitivity C-reactive protein in early radiographic knee osteoarthritis: a crosssectional study. BMJ Open 2016;6:e010763.

12 Kong L, Wang L, Meng F, et al. Association between smoking and risk of knee osteoarthritis: a systematic review and meta-analysis. Osteoarthritis Cartilage 2017;25:809-16.

13 Jung JH, Seok H, Choi SJ, et al. The association between osteoarthritis and sleep duration in Koreans: a nationwide crosssectional observational study. Clin Rheumatol 2018;37:1653-9.

14 Taylor-Gjevre RM, Gjevre JA, Nair B, et al. Components of sleep quality and sleep fragmentation in rheumatoid arthritis and osteoarthritis. Musculoskeletal Care 2011;12:n/a-159.

15 Hussain SM, Wang Y, Giles GG, et al. Female reproductive and hormonal factors and incidence of primary total knee arthroplasty due to osteoarthritis. Arthritis Rheumatol 2018;70:1022-9.

16 de Klerk BM, Schiphof D, Groeneveld FPMJ, et al. No clear association between female hormonal aspects and osteoarthritis of the hand, hip and knee: a systematic review. Rheumatology 2009;48:1160-5.

17 Miranda H, Viikari-Juntura E, Martikainen R, et al. A prospective study on knee pain and its risk factors. Osteoarthritis Cartilage 2002;10:623-30

18 Case R, Thomas E, Clarke E, et al. Prodromal symptoms in knee osteoarthritis: a nested case-control study using data from the osteoarthritis initiative. Osteoarthritis Cartilage 2015;23:1083-9.

19 Hayashi K, Mizunuma H, Fujita T, et al. Design of the Japan nurses health study: a prospective occupational cohort study of women's health in Japan. Ind Health 2007;45:679-86.

20 Nagai K, Hayashi K, Yasui T, et al. Disease history and risk of comorbidity in women's life course: a comprehensive analysis of the Japan nurses' health study baseline survey. BMJ Open 2015;5:e006360.

21 Nagin DS. Analyzing developmental trajectories: a semiparametric, group-based approach. Psychol Methods 1999;4:139-57.

22 Jones BL, Nagin DS, ROEDER K. A SAS procedure based on mixture models for estimating developmental trajectories. Sociol Methods Res 2001;29:374-93.

23 Jones BL, Nagin DS. Advances in group-based trajectory modeling and an SAS procedure for estimating them. Sociol Methods Res 2007:35:542-71.

$24 \mathrm{He}$ Y, Zaslavsky AM, Landrum MB, et al. Multiple imputation in a large-scale complex survey: a practical guide. Stat Methods Med Res 2010;19:653-70.

25 Goulston LM, Kiran A, Javaid MK, et al. Does obesity predict knee pain over fourteen years in women, independently of radiographic changes? Arthritis Care Res 2011;63:1398-406.

26 Tanamas SK, Wluka AE, Davies-Tuck M, et al. Association of weight gain with incident knee pain, stiffness, and functional difficulties: a longitudinal study. Arthritis Care Res 2013;65:34-43.

27 Macfarlane GJ, de Silva V, Jones GT. The relationship between body mass index across the life course and knee pain in adulthood: results from the 1958 birth cohort study. Rheumatology 2011:50:2251-6. 
28 Janke EA, Collins A, Kozak AT. Overview of the relationship between pain and obesity: what do we know? where do we go next? JRRD 2007;44:245-62.

29 Frilander H, Viikari-Juntura E, Heliövaara M, et al. Obesity in early adulthood predicts knee pain and walking difficulties among men: a life course study. Eur J Pain 2016;20:1278-87.

30 Vincent HK, Heywood K, Connelly J, et al. Obesity and weight loss in the treatment and prevention of osteoarthritis. Pm R 2012;4:S59-67.

31 Heo M, Allison DB, Faith MS, et al. Obesity and quality of life: mediating effects of pain and comorbidities. Obes Res 2003;11:209-16.

32 Ministry of Health, Labour and Welfare. Comprehensive survey of living conditions. 2016. Tokyo, Japan: Ministry of health, labour and welfare. Available: www.mhlw.go.jp/english/database/db-hss/cslcindex.html [Accessed 15 Aug 2019].
$33 \mathrm{Kim} \mathrm{H}$, Dropkin J, Spaeth K, et al. Patient handling and musculoskeletal disorders among hospital workers: analysis of 7 years of institutional workers' compensation claims data. Am J Ind Med 2012;55:683-90.

34 Reed LF, Battistutta D, Young J, et al. Prevalence and risk factors for foot and ankle musculoskeletal disorders experienced by nurses. BMC Musculoskelet Disord 2014;15:196.

35 Kee D, Seo SR. Musculoskeletal disorders among nursing personnel in Korea. Int J Ind Ergon 2007;37:207-12.

36 Freimann T, Coggon D, Merisalu E, et al. Risk factors for musculoskeletal pain amongst nurses in Estonia: a cross-sectional study. BMC Musculoskelet Disord 2013;14:334.

37 Smith DR, Mihashi M, Adachi Y, et al. A detailed analysis of musculoskeletal disorder risk factors among Japanese nurses. $J$ Safety Res 2006;37:195-200. 